\title{
Building Collaborative Research Teams in the Social and Behavioral Sciences
}

\author{
Chitra Rajan, Associate Vice President for Research, Iowa State University
}

$\mathrm{F}$ or the past few years, Iowa State University (ISU) has experienced severe and permanent reductions in state support. The Office for the Vice President for Research and Economic Development (VPR/ED) received its share of budget cuts and had to make some significant changes to accommodate these new economic realities. We considered and adopted a number of structural changes aimed at reducing costs. Vacant positions were not filled, contributions toward faculty start-up and retention offers were reduced, support for centers and institutes was drastically cut, and every possible 'discretionary' expense was eliminated. It should be pointed out that the VPR/ED Office relies heavily on state support, unlike our colleges who get a share of tuition revenues and have far greater fund raising opportunities.

The VPR/ED Office is responsible for many of the research support service centers (contracts and grants, compliance, animal research labs, some central research facilities, etc.) and austerity measures, if taken to an extreme, could seriously impair the university's research capabilities and assets, and harm future developments and growth. The university was very aware of this danger and did its best to protect the VPR/ED Office during these fiscally difficult years; nonetheless, budget cuts were both real and painful.

At the same time, this was a period when many of the prior investments in faculty recruitments and research infrastructures were beginning to pay off: after three years of declines in sponsored funding, the university saw not only an up-turn, but significant growth in research expenditures. Encouraged by these successes, the Office of the VPR/ED decided to continue, and in some cases even increase our support for certain critical areas, despite these difficult times. Increased support for some of the larger centers (those that had an annual research expenditure that produced over $\$ 1$ million of F\&A revenues) came in the form of sharing overhead revenues from grants; the VPR/ED Office negotiated with the Colleges and developed a plan that would return a portion of F\&A revenues back to the center so that they could invest those funds in the infrastructure necessary for their research. Such a revenue-sharing funding plan was possible because the university had adopted a decentralized budget plan whereby colleges and vice presidents received a share of the $F \& A$ revenues and were responsible for overhead costs. We also made the difficult decision to discontinue support for several centers and institutes (these centers were given the option of moving under a larger institute or into their college) so that we 
would have the resources that we needed to re-organize and truly support a smaller number of programs.

One such area was survey and behavioral studies. This is an area where ISU has considerable strength and our faculty have national recognition for their contributions to this area. Prior to the budget cuts, we had two separate centers/institutes that reported to the VPR/ED Office - the Institute for Behavioral Research (ISBR) and the Center for Survey Statistics and Methodology. Although both centers had impressive levels of external funding, the overhead costs were much greater than the indirect revenues that they were able to generate. Both centers had a large number of staff (most of them were paid from grant funds) and so were located at the university's research park, since space for such a large group was not readily available on campus. Consequently, the overhead costs - in terms of the administrative staff required to manage these two large groups and the cost of rent were very high. While both centers recognized that they had complementary and even overlapping skills and strengths, and that some economies of scale could be recognized if they worked together, the time, effort and IT resources that it would take to make that possible appeared to be prohibitive.

After three years of budget cuts, it became apparent that the amount of resources that these two centers received from the VPR/ED Office was no longer sustainable. Yet, it was important that we provided the institutional support that was necessary for research in survey methodology and behavioral sciences. Clearly, the faculty members were able to garner enough grants to cover their direct expenses; it was the overhead costs that had become prohibitive. To address this problem, we had to find a way to reduce the overhead costs. We decided to consolidate the "service" components from both centers to create a new unit - the Survey and Behavioral Research Services center (SBRS). This in itself was not particularly interesting; consolidating and merging units are not unusual cost-cutting approaches. The important question was whether the combination of the services from these two centers into one unit could provide ISU faculty with a greater level of research support than previously provided by both CSSM and ISBR?

First, the Survey and Behavioral Research Services (SBRS) was created with emphasis on providing services, not as a program or center of excellence. This meant that is primary mission was to serve as many faculty as possible, and once established, it would operate as a fee-for-service unit. If successful, it would attract enough 'business', become self-reliant (or at least, require minimal institutional funds) and therefore unaffected by fluctuations in state support for the university. To underline its service mission, the SBRS would be a unit within the university's Statistical Laboratory which was established in 1933 and part of the Statistics Department. The Statistical Laboratory is famous for the high quality statistical consulting that it has provided to ISU faculty since 1933. The Statistics Laboratory has historically specialized in statistical methods for agriculture, biology, and engineering but with less emphasis on methods for the social and behavioral sciences. The creation of 
SBRS would now give the Statistics Laboratory a chance to help faculty in this new area. The Statistics Laboratory has always reported to the VPR/ED Office and therefore the SBRS would be under its purview.

Second, the SBRS was created by merging the accounting, IT, and data collection units from the Institute for Social and Behavioral Research (ISBR) and the Center for Survey and Statistical Methodology (CSSM). To reduce costs, redundant accounting and IT positions from the ISBR/CSSM merger were eliminated; ISBR faculty affiliates were moved back to campus thereby freeing up the need for a large $(22,000 \mathrm{sq}$. ft.) space in the Research Park; and the newly merged SBRS staff were moved to a smaller $(7,000 \mathrm{sq}$. ft.) space in the Research Park. These measures reduced the overhead expenses by $50 \%$ and allowed it to offer a new slate of services as described below:

This new unit has the capacity to provide a full set of services to researchers including both proposal development and administration and all modes of data collection services.

\section{Services and Resources}

SBRS provides statistical and psychometric statistical consulting for the social and behavioral sciences to supplement the statistical consulting services of the Stat Lab. Examples of statistical methods for the social and behavioral sciences:

- Design of human subjects experiments

- Questionnaire design

- Sampling design

- Program evaluation
- Meta-analysis

- Latent variable structural equation modeling

- Psychometric reliability and validity analyses

- Assistance with SPSS

Research Consultation is available to investigators to assist in defining study objectives, identifying study populations and sample design, developing survey instruments, or evaluating appropriate survey methods. Cost estimates for conducting surveys or collaborating on proposals are supplied upon request.

Research Collaborations are supported for large surveys and projects requiring innovative survey methods. CSSM faculty provides SBRS staff with sampling and statistical methods support for grant proposals and studies using SBRS data collection services.

Grant Proposal Development and Administration Assistance are available to faculty and staff making applications for grant opportunities in all areas of research. Staff is experienced in developing budgets, coordinating proposal development and submitting proposals through the university and federal proposals systems.

Sampling Expertise for SBRS projects is provided by CSSM faculty including Random digit dialing (RDD) samples, list samples, area samples, casecontrol designs and weighting calculations.

Survey and Questionnaire Design services are provided by professional staff and include designing survey instruments, focus groups scripts, cover letters and training materials. 
Data Collection is conducted by SBRS staff in a variety of modes. The staff has experience in a wide range of data collection methods for both onetime and longitudinal surveys, including:

- Computer-assisted telephone interviewing (CATI)

- Computer-assisted personal interviewing (CAPI)

- Observational Video and Audiorecording \& coding

- Mail Surveys

- Web Surveys

- Focus Groups

Quality Control is a high priority for SBRS. All research procedures are conducted with the utmost regard for accuracy and confidentiality. Telephone interviews are reviewed on-line through a silent monitoring system to ensure high data quality standards, interviews and resulting data files are edited for accuracy, and double-entry procedures are used in key-entry tasks.

The SBRS is now a year old and its first year proved to exceed all our expectations. The unit helped faculty submit over 30 grant proposals worth about $\$ 16$ million. We have yet to see how successful these efforts will be. SBRS has 3 years to prove its viability and the associated faculty have been told that they will be assessed based on the following: (a) it has enabled research that was otherwise not possible (and not just diverted research management from other units); (b) impacted a broad group of faculty; and (c) is able to develop a "business model" that makes it fairly independent of institutional support. 